\title{
In vitro effects of different 8-methoxypsoralen treatment protocols for extracorporeal photopheresis on mononuclear cells
}

\author{
HOLGER BUDDE, ULRIKE BERNTSCH, JOACHIM RIGGERT, TOBIAS J. LEGLER
}

University Medical Center Göttingen, Göttingen, Germany

\begin{abstract}
Extracorporeal photopheresis (ECP) is an important second-line therapy for graft-versus-host disease. A central therapeutic mechanism is the induction of immune tolerance through apoptosis in patient's leukocytes, caused by ex vivo incubation with 8-methoxypsoralen (8-MOP) and subsequent UVA irradiation.

We hypothesized that different 8-MOP incubation times and an additional 8-MOP removal step could influence the apoptosis kinetics of leukocytes in general and in particular could lead to different apoptotic levels in the leukocyte subpopulations. After 8-MOP/UVA treatment of human leukocytes, cells were cultured and the percentage of annexin $V$ positive cells from several leukocyte subpopulations was determined. Only regulatory T cells (Tregs) were relatively resistant to 8-MOP/UVA induced apoptosis. When cells were incubated for 30 minutes with 8-MOP prior to UVA exposure, higher percentages of annexin $V$ positive cells were detected on day 1 and day 2 after treatment. Removal of 8-MOP after UVA exposure caused no significant changes in the apoptosis kinetics during the $72 \mathrm{~h}$ culture period compared with unwashed cells. The results of our in vitro study indicate that it could be possible to adjust the apoptosis kinetics via modulation of the 8-MOP incubation time. In further in vivo studies it should be elucidated to which extent different apoptosis kinetics influence the therapeutic effect of ECP since steady-state apoptosis levels are probably important for establishing a long lasting immune tolerance. Furthermore we found that Tregs, according to their well-known tolerogenic function, are more resistant to apoptosis after 8-MOP/UVA treatment compared to GvHD inducing $T$ cell populations.
\end{abstract}

Key words: apoptosis, regulatory T cells (Tregs), photopheresis.

(Cent Eur J Immunol 2017; 42 (1): 1-9)

\section{Introduction}

In many patients with leukemia or lymphoma, allogeneic hematopoietic stem cell transplantation (HSCT) is performed in combination with chemotherapy and sometimes radiation therapy to remove malignant cells and to prevent relapses. However, allogeneic HSCT is linked to severe side effects such as graft-versus-host disease (GvHD) which can be fatal for the patient [1]. GvHD is still one of the most challenging clinical problems after HSCT and GvHD therapy needs optimization for improved outcome.

Standard first-line therapy for GvHD is the treatment with glucocorticoids causing generalized immunosuppression. However, patients after HSCT suffer from reduced immune-competence and additional generalized immunosuppression can lead to severe opportunistic infections. Therefore, current research is focused on therapies without generalized immunosuppression such as extracorporeal photopheresis (ECP) [2]. ECP is often used as a second-line GvHD therapy and even has potential to become a part of the first-line therapy [3]. The main advantage of ECP is the induction of selective immune tolerance instead of unspecific immunosuppression [4]. After ECP therapy, no increase in opportunistic infections could be observed [5]. The procedure of ECP therapy begins with an apheresis step in which about $10 \%$ of the patient's peripheral leukocytes are harvested [6]. Subsequently, these leukocytes (mainly mononuclear cells) are incubated ex vivo with the DNA intercalator 8-methoxypsoralen (8-MOP). 8-MOP is activated by UVA light leading to DNA double-strand breaks followed by cellular apoptosis. Immediately after UVA irradiation 8-MOP returns into the inactivated stage [7]. Afterwards, treated leukocytes are reinfused into the patient.

The therapeutic effect of ECP is mediated by effects initiated by the reinfusion of apoptotic cells rather than by simple reduction of GvHD causing cytotoxic T-cells [8]. Following apoptotic cell infusion, dendritic cell differentiation via platelet activation and induction of regulatory $\mathrm{T}$ cells are important pathways leading to subsequent immune tolerance $[9,10]$. 
Cellular apoptosis is a physiological process inducing immune tolerance in contrast to inflammation causing necrosis [11]. However, tolerance induction is a dose-dependent process and too much or too little apoptosis is limiting the therapeutic benefit [12]. In clinical protocols, about $10 \%$ of the peripheral leukocytes are commonly used for a single ECP procedure. However, recent publications suggest that lower concentrations of ECP treated cells are also effective [13]. Beside considerations about the optimal ECP cell dose, apoptosis kinetics is probably another important factor. If too many apoptotic cells have to be removed by the monocyte/macrophage system, inflammatory pathways are activated and tolerance induction might not occur [12]. Furthermore, a more constant application of apoptotic cells is perhaps required to establish and maintain a tolerogenic effect [14].

Based on these findings, different questions arise for the identification of the most effective ECP protocol. For example, since ECP is based on an apheresis procedure which is a physical effort for patients and requires a sufficient venous access, it cannot be performed as often as pharmaceutical therapy. Usually ECP initiated for the treatment of acute GvHD is performed at weekly intervals on one or two consecutive days $[15,16]$. As apoptosis is an important ECP mechanism one might speculate that a continuous supply and subsequent degradation of apoptotic cells would more effectively establish immune tolerance compared with the weekly therapy.

We hypothesize that although apoptosis of the treated cell product is desirable, apoptosis of all cells should not occur immediately after treatment but be more delayed over the first days to achieve a prolonged or more constant level of apoptotic cells. The central aim of our study was to find out whether the time-dependent increase in the apoptosis level of different leukocyte subpopulations in vitro can be influenced by different 8-MOP/UVA treatment protocols.

\section{Material and methods}

\section{Blood samples}

All blood samples were obtained from healthy blood donors who regularly attend the blood donation service of the University Medical Center Göttingen. Altogether 36 donors aged 18-65 years were included in the study. For each treatment condition buffy-coats from nine different wholeblood donations were used. All donors gave written consent that a part of their blood donation can be used for scientific purposes and procedures were performed in accordance with the Declaration of Helsinki. The study was approved by the local ethics committee of the University Medical Center Göttingen (no. 18/6/12).

Mononuclear cells were enriched by Ficoll density-gradient-centrifugation with Ficoll-Paque Plus (GE Healthcare Biosciences AB, Uppsala, Sweden). Coagulation of blood samples was prevented by sodium-citrate. Cells were sepa- rated by centrifugation at $600 \mathrm{~g}$ for 20 minutes followed by two platelet depletion steps with $100 \mathrm{~g}$ for 10 minutes each.

\section{8-MOP and UVA treatment}

A liquid solution of 8-MOP was produced by the pharmaceutical department of the University Medical Center Göttingen. Leukocytes were suspended in $25 \mathrm{~cm}^{2}$ flasks in $5 \mathrm{ml}$ phosphate-buffered saline (PBS; Lonza, Verviers, Belgium), incubated with $0.2 \mu \mathrm{g} / \mathrm{ml}$ 8-MOP and exposed to UVA light $\left(2 \mathrm{~J} / \mathrm{cm}^{2}\right)$. Four different conditions were compared: in the first condition cells were incubated for 30 min with 8-MOP prior to UVA exposure. Following UVA exposure, cells were washed once with PBS and centrifuged at $500 \mathrm{~g}$ for $5 \mathrm{~min}$ for 8-MOP removal. In the second condition, 8-MOP was given to the cell suspension immediately before UVA exposure without pre-incubation. Subsequently, cells were washed for 8-MOP removal in the same manner as in the first condition. In the third condition, 8-MOP was added immediately before UVA exposure like in the second condition but cells were resuspended after UVA exposure in culture medium without any additional washing step with PBS. After 8-MOP and UVA treatment, cells of all treatment groups were cultured with RPMI-1640 medium (Gibco, Paisley, UK), 10\% fetal calf serum (Gibco, NY, USA) and $1 \%$ penicillin/streptomycin (Gibco, NY, USA) at $37^{\circ} \mathrm{C}$ and $5 \% \mathrm{CO}_{2}$. In the control group, cells were cultured immediately after Ficoll separation without any 8-MOP or UVA treatment.

\section{Flow cytometry}

Cells of all subgroups were stained with fluorescent antibodies for different leukocyte antigens immediately after 8-MOP/UVA treatment (day 0) and in addition after 1, 2 and 3 days in culture. Fc receptors were blocked for $10 \mathrm{~min}$ at room temperature (Human TruStain FcX, BioLegend, San Diego, CA, USA). Anti-human CD45 (PE, Clone 2D1, Mouse IgG1), CD4 (eFluor450, Clone RPA-T4, Mouse IgG1), CD8 (PE, Clone SK1, Mouse IgG1) and CD25 (APC, Clone BC96, Mouse IgG1) were purchased from eBioscience, San Diego, CA, USA. Anti-human CD3 (PE, Clone HIT3a, Mouse IgG2a) was purchased from BD Pharmingen (San Diego, CA, USA) and CD14 (APC, Clone M5E2, Mouse IgG2a), CD19 (APC, Clone HIB19, Mouse IgG1) and CD56 (APC, Clone HCD56, Mouse IgG1) from BioLegend (San Diego, CA, USA). After staining of CD-antigens on the cell surface, cellular apoptosis was detected with annexin V. For annexin V-staining, cells were resuspended in annexin V Binding Buffer (BioLegend) and incubated with FITC-labeled annexin V (BioLegend) for 10 minutes at room temperature prior to flow cytometry analysis (FACS Canto II, BD Biosciences, San Jose, CA, USA). Regulatory T cells were defined as CD4+ CD25+ cells without performing FoxP3 staining, because intracellular FoxP3 binding needs permeabilization of the cell membrane which 
is not compatible with the annexin $\mathrm{V}$ assay. Even though Tregs are identified in most of the current studies by CD4+ CD25+ FoxP3+ triple staining, different studies show that in general $90-97 \%$ of all CD4+ CD25+ cells are FoxP3 positive $[17,18]$. Analysis of flow cytometry data was performed using the FACSDiva Software (BD Biosciences).

\section{Statistics}

In order to analyze the effects of culture duration and 8-MOP/UVA treatment condition on the apoptosis levels of the corresponding leukocyte subpopulations, two-way repeated measures analysis of variance (ANOVA) were performed. Subsequent to the ANOVA, subgroups were analyzed with Tukey's post-hoc test. $P$-values $<0.05$ were defined as sta- tistically significant. Figures are shown as mean + SEM and significant $p$-values were indicated above the bars.

\section{Results}

\section{8-MOP/UVA treatment causes apoptosis in all leukocyte subpopulations}

Since steady-state levels of apoptotic cells are known to induce immune tolerance, we wanted to investigate whether changes in the 8-MOP/UVA protocol can influence the kinetics of apoptosis induction in the treated leukocytes. In all treatment groups we observed a continuous increase in annexin $\mathrm{V}$ positive cells during the culture pe- $\square$ ctrl.

- 30 min preincub. + wash

$\square$ no preincub. + wash

$\square$ no preincub. w/o wash
A

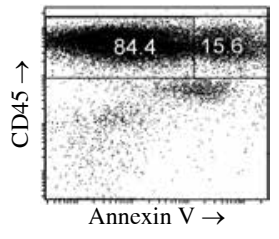

B

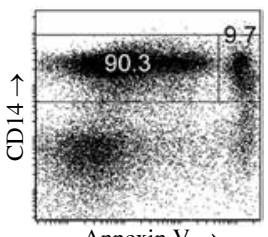

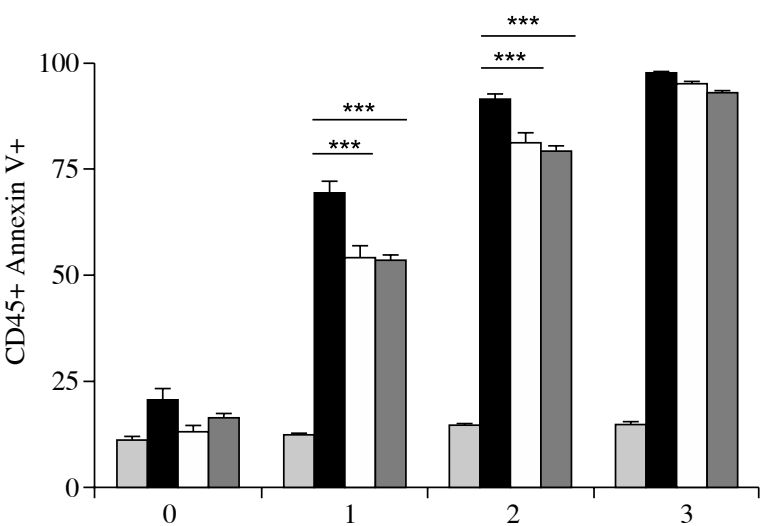

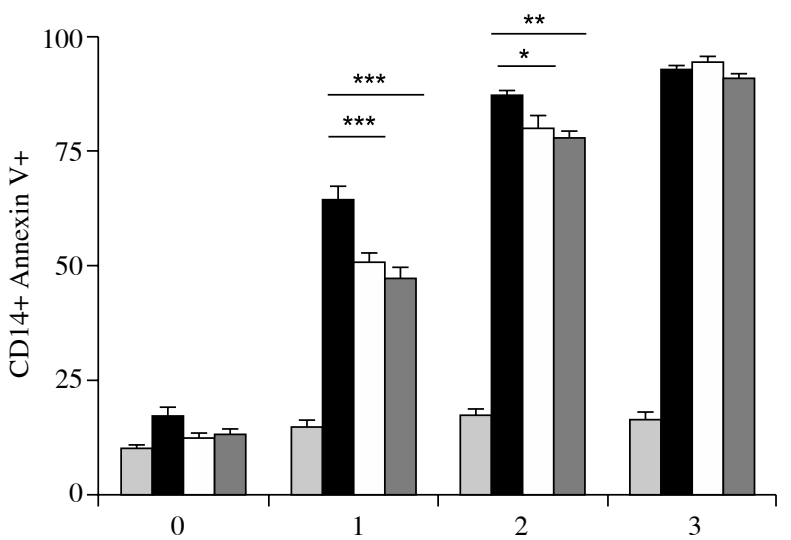

Fig. 1. Apoptosis of leukocytes and monocytes after different 8-MOP/UVA treatments. Leukocytes were stained for the pan leukocyte marker CD45 and for annexin V as apoptosis marker and analyzed by flow cytometry (A). Monocytes were detected by CD14 staining (B). Percentages of annexin V positive cells out of all CD45 or CD14 cells are shown in the bar chart. Treatment conditions for Figs. 1-3: Without treatment (ctrl.), with 30 minutes' 8-MOP pre-incubation and subsequent washing step for 8-MOP removal before culturing (30 min pre-incubation + wash), with 8-MOP addition immediately before UVA irradiation and subsequent 8-MOP removal before culturing (no pre-incubation + wash) and with 8-MOP addition immediately before UVA irradiation without 8-MOP removal before culturing (no pre-incubation w/o wash). Apoptosis of untreated ctrl. samples after 1, 2 and 3 days in culture was always significantly lower $(p<0.001)$ compared with treated samples (Figs. 1-3). Other significant differences are depicted with $* p<0.05$, ** $p<0.01$ or $* * * p<0.001$ 
A

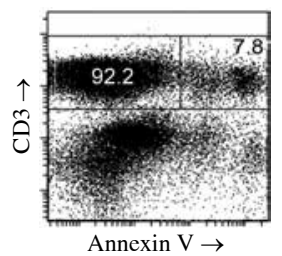

B

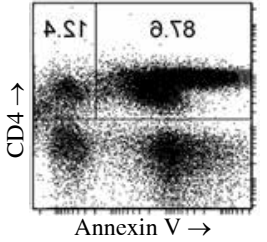

C

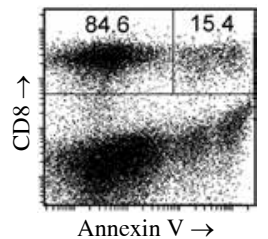

D

\section{$\square$ ctrl.}

- 30 min preincub. + wash

$\square$ no preincub. + wash

口no preincub. w/o wash
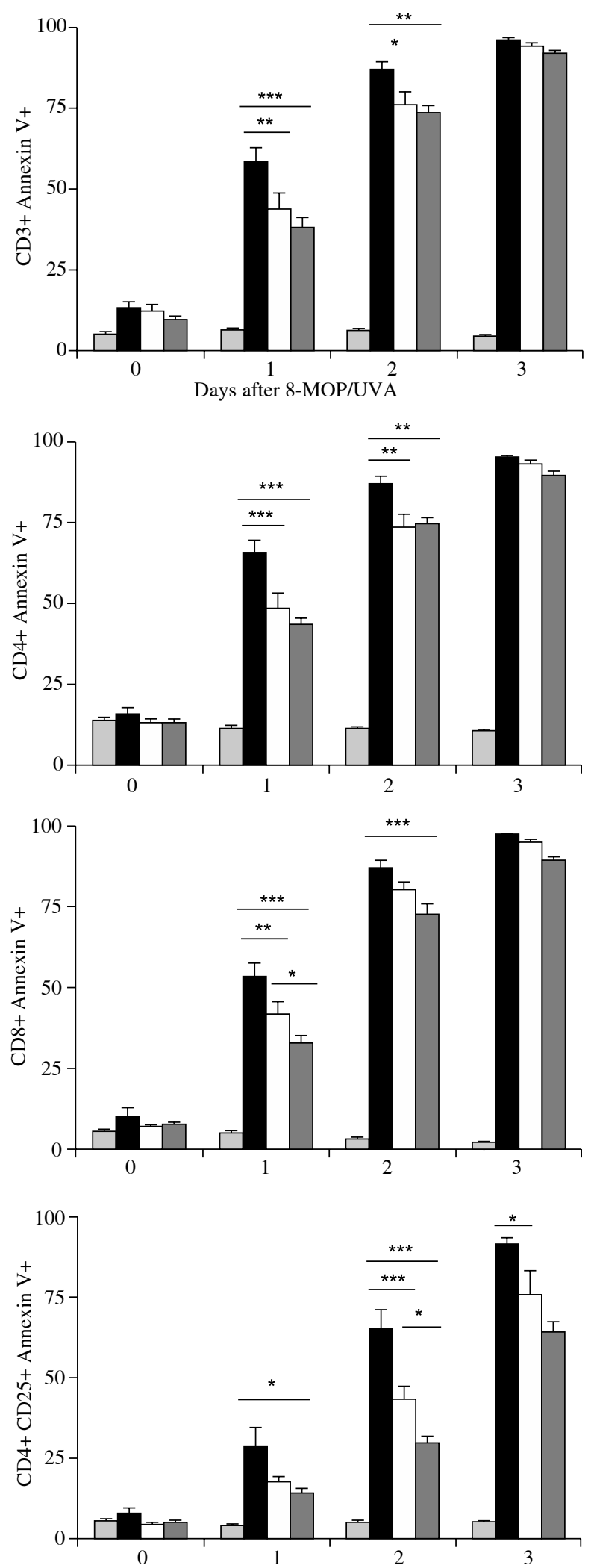

Fig. 2. Apoptosis of $\mathrm{T}$ cells after different 8-MOP/UVA treatments. Leukocytes were stained with $\mathrm{CD} 3$ as pan $\mathrm{T}$ cell marker (A), CD4 as T helper cell marker (B), CD8 as cytotoxic T cell marker (C) and with CD4 CD25 co-staining as regulatory $\mathrm{T}$ cell marker (D). Percentages of annexin $\mathrm{V}$ positive cells out of all cells from the corresponding cell type are shown in the bar charts. For treatment conditions, see legend of Fig. 1 
A

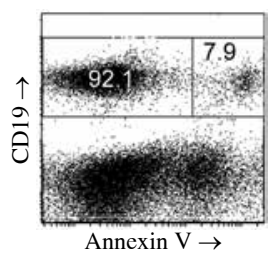

B

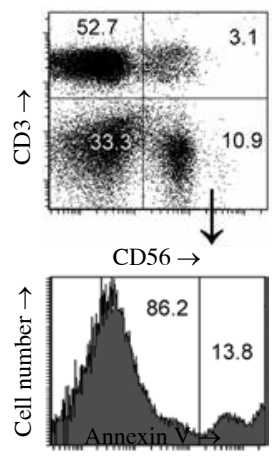

C

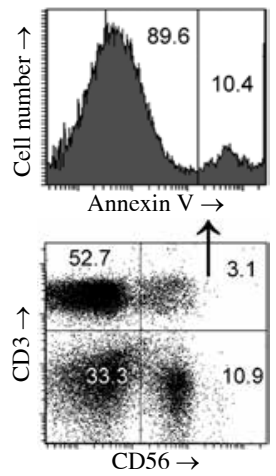


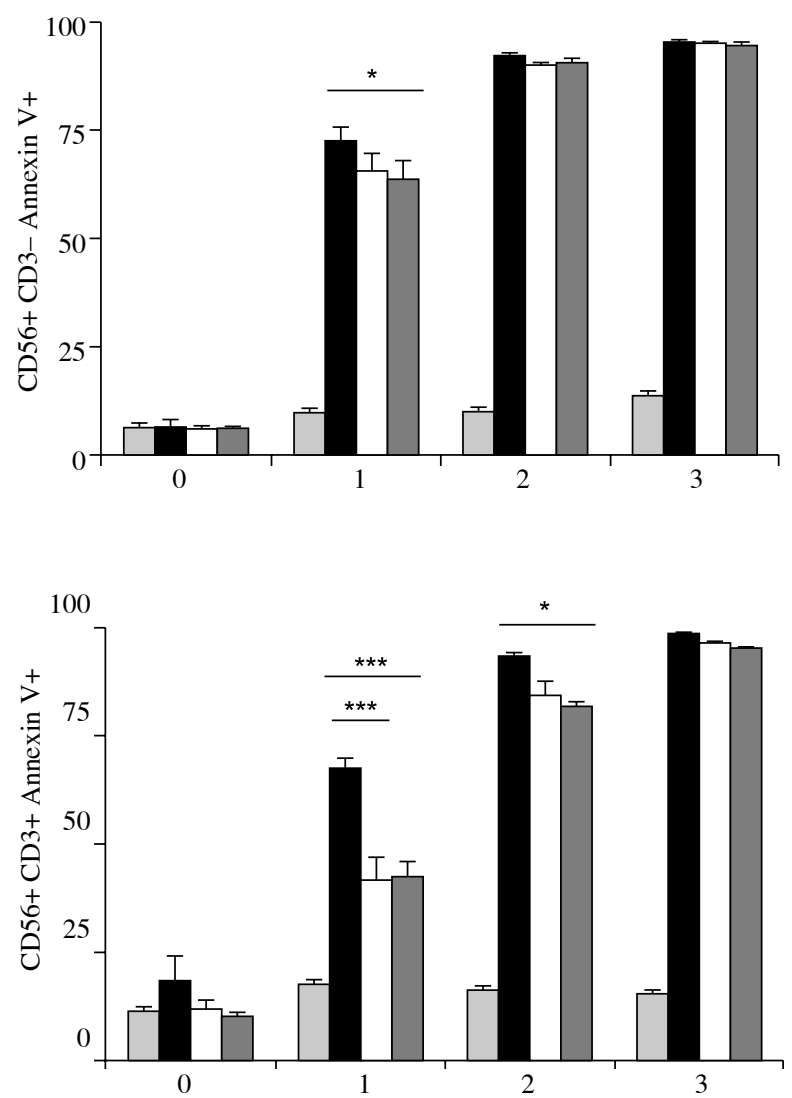

Fig. 3. Apoptosis of B cells, NK cells and NKT cells after different 8-MOP/UVA treatments. B cells were identified with marker CD19 (A). NK cells were defined as CD56 positive and CD3 negative cells (B) and NKT cells as CD56 CD3 double-positive cells (C). Percentages of annexin V positive cells out of all cells from the corresponding cell type are shown in the bar charts. For treatment conditions, see legend of Fig. 1

riod of 3 days (Figs. 1-3). In the untreated controls we could not observe considerably increased apoptosis levels during the 3-day culture period (Figs.1-3, day 0). The first quantification of annexin $\mathrm{V}$ positive cells was performed immediately after 8-MOP/UVA treatment before resuspension in cell culture medium. Compared to controls, a small increase in annexin $\mathrm{V}$ positive cells immediately after treatment occurred only in the CD3+ T cell and the CD14+ monocyte population (Figs. 1B, 2A). In the other 8-MOP/UVA treated leukocyte subpopulations, apoptosis levels at this early time point were not statistically different compared to control samples (Figs. 1-3). In the follow- 
Table 1. Two-way repeated measures ANOVA for analysis of differences between time points and treatment protocols. $P$ - and $F$-values and the degree of freedom (D.F.) are shown for the analysis of changes over time (day $0,1,2$ and 3 ) and differences in the 3 experimental 8-MOP/UVA treatment protocols

\begin{tabular}{ccccccc}
\hline Cell-Type & \multicolumn{2}{c}{$\begin{array}{c}\text { Differences between time points } \\
\text { (day 0, 1, 2, 3) }\end{array}$} & \multicolumn{2}{c}{$\begin{array}{c}\text { Differences between the } \\
\text { 3 treatment protocols }\end{array}$} \\
\cline { 2 - 7 } & $\boldsymbol{P}$-value & $\boldsymbol{F}$-value & D.F. & $\boldsymbol{P}$-value & $\boldsymbol{F}$-value & D.F. \\
\hline CD45+ & $<0.0001$ & 1857 & 3 & $<0.0001$ & 16,0 & 2 \\
\hline CD14+ & $<0.0001$ & 1575 & 3 & 0.0002 & 12,0 & 2 \\
\hline CD3+ & $<0.0001$ & 880 & 3 & 0.0071 & 6,1 & 2 \\
\hline CD4+ & $<0.0001$ & 685 & 3 & 0.0009 & 9,6 & 2 \\
\hline CD8+ & $<0.0001$ & 1143 & 3 & 0.0016 & 8,5 & 2 \\
\hline CD4+ CD25+ & $<0.0001$ & 341 & 3 & 0.0003 & 11,5 & 2 \\
\hline CD19+ & $<0.0001$ & 2266 & 3 & 0.0008 & 9,8 & 2 \\
\hline CD3- & $<0.0001$ & 1082 & 3 & $n .5$ & 2,1 & 2 \\
\hline CD56+ & & & & $0.14)$ & & 2 \\
\hline CD3+ CD56+ & $<0.0001$ & 652 & 3 & 0.0005 & 10,5 & 2 \\
\hline
\end{tabular}

ing 3 days after 8-MOP/UVA treatment, apoptosis levels increased continuously. On day 1 most cell populations showed already around $50 \%$ apoptosis and reached in between 90 and $99 \%$ on day 3 independently of the treatment protocol (Figs. 1-3).

\section{8-MOP pre-incubation for $\mathbf{3 0}$ minutes leads to more rapid apoptosis}

The three different 8-MOP/UVA treatment protocols showed significant differences in their apoptosis patterns (Table 1). The highest increase in apoptosis levels occurred in all cell populations when cells were pre-incubated for 30 min with 8-MOP prior to UVA exposure (Figs. 1-3). Directly after 8-MOP/UVA treatment we already observed in several cell populations higher percentages for apoptosis $(6-21 \%)$ compared to untreated cells (5-14\%, Figs. $1-3)$. However, the difference at this early time point was statistically significant only for CD3+ T-cells $(p<0.05$, Fig. 2A) and CD14+ monocytes ( $p<0.05$, Fig. 1B). Substantial mean apoptosis levels of 53 to $73 \%$ were detected 1 day after using the 30-min 8-MOP pre-incubation protocol in CD45+ cells and in most leukocyte subpopulations such as CD3+, CD4+, CD8+, CD56+ CD3-, CD19+ and CD14+ cells (Figs. 1-3). In contrast, apoptosis levels of cells cultured for 1 day with the two other protocols without 30-min 8-MOP pre-incubation were in almost all cases significantly lower $(p<0.05)$, depicted by mean apoptosis levels of $37-58 \%$. The difference in the apoptosis rate was still statistically significant after 2 days in most leukocyte subpopulations. Only NK-cells showed high apoptosis levels 1 day after 8-MOP/UVA treatment independent of the three different treatment protocols (Fig. 3B).

\section{8-MOP depletion before cell-culturing does not reduce apoptosis}

Furthermore, we investigated whether the final washing step for 8-MOP removal before culturing has an impact on the apoptosis rate of the leukocyte subpopulations. We compared two conditions, the first one with a PBS washing step for 8-MOP removal and the second one without the final washing step in which 8-MOP remained in the cell suspension during the 3 -day culture period. In both conditions 8-MOP was added to the cells immediately before UVA exposure. Comparing both conditions with and without residual 8-MOP during the 3-day culture period in most cell types we observed a tendency for slightly lower apoptosis levels in the condition without 8-MOP removal before culturing (Figs. 1-3). However, no statistically significant differences appeared in cellular apoptosis at any time point. As mentioned above, nearly complete apoptosis was detected after 3 days of cell culture in most leukocyte subpopulations independent of the treatment protocol except in CD4+ CD25+ Tregs (Fig. 2D).

\section{Tregs are more resistant to 8-MOP/UVA treatment}

The apoptosis kinetics of most leukocyte subpopulations were similar after 8-MOP/UVA treatment: on day 1 we found mean apoptosis levels of $33-75 \%$ depending on the cell subpopulation and the treatment protocol (Figs. 1-3). In contrast to all other cell populations, after 1 day CD4+ CD25+ Tregs showed very low mean apoptosis levels of only 14-29\% depending on the different treatment conditions (Fig. 2D). When compared with all other cell 
types, apoptosis of Tregs was significantly lower in each of the three treatment conditions $(p<0.001)$. Furthermore, after 2 and 3 days in cell culture, the Treg apoptosis rate was lower as compared to the other leukocyte subpopulations. Treg apoptosis after 2 days ranged from 30 to $65 \%$ compared with 73 to $93 \%$ in the other leukocyte subpopulations $(p<0.01)$. Even on day 3, Treg apoptosis was less complete (64 to $92 \%$ ) than in other cell populations (90 to $99 \%, p<0.001)$. Especially in the treatment with the clinically applied protocol, in which UVA irradiation is performed directly after the addition of 8-MOP (without 30 min 8-MOP pre-incubation) and without final 8-MOP removal, Tregs showed a relatively low mean apoptosis rate of $64 \%$ on day 3 ( $p<0.001$ vs. all other cell populations).

\section{High apoptosis rates in $B$ cells and NK cells already one day after 8-MOP/UVA treatment}

In contrast to low apoptosis levels in Tregs, in NK and $\mathrm{B}$ cells the highest apoptosis rates of all leukocyte subpopulations were found (Figs. 3A, B). Apoptosis levels in NK cells were 64 to $73 \%$ already one day after treatment. In addition, NK and B cells showed high apoptosis levels with $90-92 \%$ and $90-95 \%$, respectively, 2 days after 8-MOP/UVA treatment whereas such a high apoptosis rate was not observed until day 3 in the other leukocyte subpopulations $(p<0.01)$. Interestingly, these high apoptosis rates on day 2 were observed even if 8 -MOP was added to the cell suspension immediately before UVA irradiation without the $30 \mathrm{~min}$ pre-incubation step.

\section{Discussion}

Controlled removal of apoptotic leukocytes is assumed to be one of the main mechanisms of ECP therapy. The resulting induction of immune tolerance is most probably dependent on a defined level of apoptotic cells [14]. This general mechanism of selective immunologic silencing has a considerable potential for treatment of diseases with a hyper-reactive immune system. Since apoptosis of leukocytes through ECP therapy is dependent on 8-MOP and UVA treatment, the corresponding protocols should have a crucial impact on the quantity and time-dependent occurrence of apoptotic cells. Interestingly, clinical treatment protocols differ from experimental animal models. In clinical protocols 8-MOP is added immediately before UVA exposure whereas in ECP mouse models 8-MOP is often pre-incubated for $30 \mathrm{~min}$ before UVA exposure [19, 20]. Another difference is that in clinical protocols 8-MOP is not removed from the leukocyte suspension after UVA exposure in general, but in many published mouse experiments 8-MOP removal is performed before injection of the treated leukocytes into the animals [20-23]. One reason for this difference is the in vivo toxicity of 8-MOP in mice, which is why 8 -MOP should be removed before injection of the treated cells $[24,25]$. In the clinical setting, the 8-MOP concentration used in the ECP therapy is well tolerated without removing 8-MOP from the cell suspension. Therefore, one should keep in mind that these different protocols could result in different apoptosis patterns with different therapeutic outcomes. Hence the aim of our study was to compare the apoptosis characteristics of several leukocyte subpopulations after treatment with different 8-MOP/UVA protocols.

In concordance with results from other studies we found that 8 -MOP/UVA treatment led to almost complete apoptosis [9, 26, 27]. In our experiments we found that apoptosis levels increased successively with time after treatment and were almost complete after 3 days independent of the 8-MOP/UVA treatment protocol. However, the different protocols we used showed different apoptosis kinetics. The strongest increase in apoptosis was observed when cells were pre-incubated with 8-MOP $30 \mathrm{~min}$ prior to UVA irradiation. Although, Karolak et al. found that 8-MOP uptake from supernatant to lymphocyte cytoplasm reached equilibrium after two minutes, there is possibly another time for uptake into the nucleus and stable incorporation into the DNA double-strands [28]. Probably after $30 \mathrm{~min}$ of pre-incubation 8-MOP is able to incorporate into the DNA more completely and intracellular caspase activation is better induced by higher fractions of damaged DNA [29]. The washing step for 8-MOP removal did not result in significant changes in the apoptosis kinetic. On the one hand this result was expected, since it has been shown in previous studies that 8 -MOP returns to an inactive state when UVA light irradiation is stopped [7], but on the other hand the experiment was important since different procedures such as the addition of washing and centrifugation steps immediately after 8-MOP/UVA treatment implies additional centrifugation stress and time before bringing the cells in culture. Having a closer look at our results, it is visible in the condition with the additional washing step that the apoptosis levels are slightly, although not significantly, higher than in the condition without the washing step.

Since immune tolerance can be efficiently induced by a steady-state level of apoptotic cells, the conventional protocol from clinics seems to be superior to other protocols e.g. used in animal experiments. In the clinical protocol, apoptosis of the corresponding cell populations is more equally distributed over the first $72 \mathrm{~h}$ after PUVA treatment. Therefore the apoptosis level is more continuous than in protocols with 8-MOP pre-incubation leading to a fast increase in apoptosis directly after 8-MOP/UVA treatment.

The main cause for GvHD onset are alloreactive $\mathrm{T}$ cells arising from the hematopoietic graft. We could demonstrate that CD3+ T cells in general and CD4+ as well as CD8+ T cells in particular undergo almost complete apoptosis within $72 \mathrm{~h}$ after 8-MOP/UVA treatment in vitro. This effect was very robust and independent of the 
variations in the protocols. Our findings are further confirmed by the study of Schmid et al. showing an increase in annexin $\mathrm{V}$ positive $\mathrm{CD} 3 \mathrm{~T}$ cells after ex vivo $\mathrm{ECP}$ with high final apoptosis levels after $72 \mathrm{~h}$ in vitro [30].

The different leukocyte populations showed similar apoptosis characteristics after 8-MOP/UVA treatment. Only in the CD4+ CD25+ fraction we found a different apoptosis pattern. Since $90-97 \%$ of the CD4+ CD25+ cell population is expressing the FoxP3 Treg transcription factor $[9,10]$, we suggest that these apoptosis pattern reflects the apoptosis of the Treg population. The increase in Treg cell death as well as the final apoptosis levels $72 \mathrm{~h}$ after 8-MOP/UVA treatment were smaller than in all other cell populations. Lowest Treg apoptosis was reached by the clinical protocol without 30-min 8-MOP pre-incubation. Therefore, the clinical protocol seems to be more favorable for maintaining Tregs over the ECP therapy. Since Tregs are most probably the central effector cells in ECP-mediated induction of immune tolerance, the effect probably contributes to the therapeutic mechanisms of ECP treatment by shifting the T cell ratio from T-helper and cytotoxic $\mathrm{T}$ cells to the Treg fraction [31]. This finding matches observations from different other studies, in which increased numbers of Tregs after ECP therapy were found $[23,32,33]$.

Contributing to the discussion whether ECP is able to induce monocyte apoptosis or not, we found that monocytes undergo apoptosis after 8-MOP/UVA treatment [34, 35]. Their apoptosis kinetics were similar to most other leukocyte subpopulations and final monocyte apoptosis after $72 \mathrm{~h}$ in vitro was also above $90 \%$. In addition Hannani et al. observed that the migratory behavior of monocytes is altered directly after ECP treatment [34]. Interestingly, they found that even after induction of apoptosis in monocytes, the ability of cytokine secretion was not impaired in the first $24 \mathrm{~h}$.

In two leukocyte subpopulations we found very high susceptibility to 8-MOP/UVA mediated apoptosis. B cells as well as NK cells showed almost complete apoptosis already after $48 \mathrm{~h}$ in vitro. B cell apoptosis is probably another factor contributing to the therapeutic effect of ECP, since B cell depletion through administration of anti-CD20 antibody Rituximab was found to be preventive for developing chronic GvHD after HSCT [36, 37]. On the other hand, NK cell reduction may not contribute to the positive ECP effects, because NK cells may have potential in modulating alloreactive immune-responses and alleviation of GvHD [38]. NK cell apoptosis could be therefore a side effect with less impact than the other positive cellular effects generated by ECP.

In conclusion, we found that leukocyte apoptosis kinetics can be influenced by variation of 8-MOP (pre-)incubation time and, on a small scale, by the final washing step for 8-MOP removal after treatment. Most cell populations show a strong apoptosis response with apoptosis levels more than $90 \%$ after $72 \mathrm{~h}$ in vitro. In particular, cytotoxic $\mathrm{T}$ cells and T-helper cells as well as B cells, which are the main drivers in graft-versus-host alloreaction, undergo almost complete apoptosis. In contrast, immune tolerance mediating Tregs are more resistant to 8-MOP/UVA induced apoptosis. The most interesting protocol with the highest Treg survival-rate and rather more prolonged or steady-state apoptosis levels of the other leukocyte populations was found to be 8-MOP/UVA incubation only during UVA exposure without 8-MOP pre-incubation and without final 8-MOP removal. Future in vivo animal or clinical studies are required to elucidate the influence of different apoptosis kinetics on the outcome of the ECP therapy.

The authors declare no conflict of interest.

\section{References}

1. Blazar BR, Murphy WJ, Abedi M (2012): Advances in graftversus-host disease biology and therapy. Nat Rev Immunol 12: 443-458.

2. Bluestone JA, Auchincloss H, Nepom GT, et al. (2010): The Immune Tolerance Network at 10 years: tolerance research at the bedside: Nat Rev Immunol 10: 797-803.

3. Merlin E, Paillard C, Rochette E, et al. (2010): Extracorporeal photochemotherapy as second- or first-line therapy of acute GVHD? Bone Marrow Transplant 45: 963-965.

4. Marshall SR (2006): Technology insight: ECP for the treatment of GvHD-can we offer selective immune control without generalized immunosuppression? Nat Clin Pract Oncol 3: 302-314.

5. Suchin KR, Cassin M, Washko R, et al. (1999): Extracorporeal photochemotherapy does not suppress T- or B-cell responses to novel or recall antigens. J Am Acad Dermatol 41: 980-986.

6. Wolf CE, Meyer M, Riggert J (2005): Leukapheresis for the extraction of monocytes and various lymphocyte subpopulations from peripheral blood: product quality and prediction of the yield using different harvest procedures. Vox Sang 88: 249-255.

7. Heshmati F (2003): Mechanisms of action of extracorporeal photochemotherapy. Transfus Apher Sci 29: 61-70.

8. Voss CY, Fry TJ, Coppes MJ, et al. (2010): Extending the horizon for cell-based immunotherapy by understanding the mechanisms of action of photopheresis. Transfus Med Rev 24: $22-32$.

9. Durazzo TS, Tigelaar RE, Filler R, et al. (2014): Induction of monocyte-to-dendritic cell maturation by extracorporeal photochemotherapy: initiation via direct platelet signaling. Transfus Apher Sci 50: 370-378.

10. Lamioni A, Parisi F, Isacchi G, et al. (2005): The immunological effects of extracorporeal photopheresis unraveled: induction of tolerogenic dendritic cells in vitro and regulatory T cells in vivo. Transplantation 79: 846-850.

11. Jiang H, Lu Z, Pan S, et al. (2006): Opposite effects of donor apoptotic versus necrotic splenocytes on splenic allograft tolerance. J Surg Res 136: 247-254.

12. Grodzicky T, Elkon KB (2002) Apoptosis: a case where too much or too little can lead to autoimmunity. Mt Sinai J Med 69: 208-219. 
13. Hackstein H, Amoros JJ, Bein G, et al. (2014): Successful use of miniphotopheresis for the treatment of graft-versus-host disease. Transfusion 54: 2022-2027.

14. Birge RB, Ucker DS (2008): Innate apoptotic immunity: the calming touch of death. Cell Death Differ 15: 1096-1102.

15. Alcindor T, Gorgun G, Miller KB, et al. (2001): Immunomodulatory effects of extracorporeal photochemotherapy in patients with extensive chronic graft-versus-host disease. Blood 98: 1622-1625.

16. Perfetti P, Carlier P, Strada P, et al. (2008): Extracorporeal photopheresis for the treatment of steroid refractory acute GVHD. Bone Marrow Transplant 42: 609-617.

17. Porto G, Giordano RJ, Marti LC, et al. (2011): Identification of novel immunoregulatory molecules in human thymic regulatory CD4+CD25+ T cells by phage display. PLoS One 6 : e21702.

18. Chen X, Subleski JJ, Hamano R, et al. (2010): Co-expression of TNFR2 and CD25 identifies more of the functional CD4+FOXP3+ regulatory T cells in human peripheral blood. Eur J Immunol 40: 1099-1106.

19. Ward DM (2011): Extracorporeal photopheresis: how, when, and why. J Clin Apher 26: 276-285.

20. Gatza E, Rogers CE, Clouthier SG, et al. (2008): Extracorporeal photopheresis reverses experimental graft-versus-host disease through regulatory T cells. Blood 112: 1515-1521.

21. Florek M, Sega EI, Leveson-Gower DB, et al. (2014): Autologous apoptotic cells preceding transplantation enhance survival in lethal murine graft-versus-host models. Blood 124: 1832-1842.

22. Budde H, Kolb S, Salinas Tejedor L, et al. (2014): Modified extracorporeal photopheresis with cells from a healthy donor for acute graft-versus-host disease in a mouse model. PLoS One 9: e105896.

23. Maeda A, Schwarz A, Bullinger A, et al. (2008): Experimental extracorporeal photopheresis inhibits the sensitization and effector phases of contact hypersensitivity via two mechanisms: generation of IL-10 and induction of regulatory T cells. J Immunol 181: 5956-5962.

24. Apostolou A, Williams RE, Comereski CR (1979): Acute toxicity of micronized 8-Methoxypsoralen in rodents. Drug Chem Toxicol 2: 309-313.

25. Dubertret L, Averbeck D, Zajdela F, Bisagni E, Moustacchi E, Touraine R, Latarjet R (1979): Photochemotherapy (PUVA) of psoriasis using 3-carbethoxypsoralen, a non-carcinogenic compound in mice. Br J Dermatol 101: 379-389.

26. Gasparro FP, Dall'Amico R, Goldminz D, et al. (1989): Molecular aspects of extracorporeal photochemotherapy. Yale J Biol Med 62: 579-593.

27. Bladon J, Taylor PC (2006): Extracorporeal photopheresis: a focus on apoptosis and cytokines. J Dermatol Sci 43: 85-94.

28. Karolak L, Tod M, Leon A, et al. (1992): In vitro kinetics of 8-methoxypsoralen penetration into human lymphoid cells. Photodermatol Photoimmunol Photomed 9: 58-60.

29. Roos WP, Kaina B (2006): DNA damage-induced cell death by apoptosis. Trends Mol Med 12: 440-450.

30. Schmid D, Grabmer C, Streif D, et al. (2015): T-cell death, phosphatidylserine exposure and reduced proliferation rate to validate extracorporeal photochemotherapy. Vox Sang 108: $82-88$.

31. Xia CQ, Campbell KA, Clare-Salzler MJ (2009): Extracorporeal photopheresis-induced immune tolerance: a focus on modulation of antigen-presenting cells and induction of regu- latory T cells by apoptotic cells. Curr Opin Organ Transplant 14: 338-343.

32. Lorenz K, Rommel K, Mani J, et al. (2015): Modulation of lymphocyte subpopulations by extracorporeal photopheresis in patients with acute graft-versus-host disease or graft rejection. Leuk Lymphoma 56: 671-675.

33. Di Biaso, Di Maio L, Bugarin C, et al. (2009): Regulatory $\mathrm{T}$ cells and extracorporeal photochemotherapy: correlation with clinical response and decreased frequency of proinflammatory T cells. Transplantation 87: 1422-1425.

34. Hannani D, Gabert F, Laurin D, et al. (2010): Photochemotherapy induces the apoptosis of monocytes without impairing their function. Transplantation 89: 492-499.

35. Tambur AR, Ortegel JW, Morales A, et al. (2000): Extracorporeal photopheresis induces lymphocyte but not monocyte apoptosis. Transplant Proc 32: 747-748.

36. Cutler C, Kim HT, Bindra B, et al. (2013): Rituximab prophylaxis prevents corticosteroid-requiring chronic GVHD after allogeneic peripheral blood stem cell transplantation: results of a phase 2 trial. Blood 122: 1510-1517.

37. Alousi AM, Uberti J, Ratanatharathorn V (2010): The role of B cell depleting therapy in graft versus host disease after allogeneic hematopoietic cell transplant. Leuk Lymphoma 51: 376-389.

38. Palmer JM, Rajasekaran K, Thakar MS, et al. (2013): Clinical relevance of natural killer cells following hematopoietic stem cell transplantation. J Cancer 4: 25-35. 